\title{
Tax Enforcement Problems
}

\section{Francisco Marhuenda and Ignacio Ortuño-Ortín*}

\author{
University of Alicante, ES-03080 Alicante, Spain
}

\begin{abstract}
We study an income tax enforcement problem using a principal-agent model where the government sets the tax and inspection functions. These are announced to the agents and there is no commitment problem. The penalty function for dishonest taxpayers is given exogenously and satisfies certain social norms. We prove that, for a large family of penalty functions, this policy is such that honesty implies regressiveness. This result does not depend on the fact that agents know the true probability of inspection.
\end{abstract}

\section{Introduction}

The fact that agents in an economy have private information sets important constraints on the kind of policies a government can achieve. An example of this phenomenon is the optimal income taxation problem where agents differ in their abilities to transform labor into income. The government cannot observe this ability and such an asymmetry of information restricts its policies as compared with the ideal case where it has complete information.

We study the case in which the government knows the distribution of income of the population but can only know the income of each specific agent by carrying out a perfect, but costly, tax inspection. In general, only a fraction of the agents will be audited, thereby creating the possibility of tax evasion. The tax paid by an agent depends on her own income level report. In addition, a penalty is imposed on those agents who are found to be dishonest. The problem faced by the government is to find the optimal tax schedule, taking into account the enforcement costs associated with it.

The enforcement problem becomes trivial if there are no bounds on the admissible penalties: the government would audit all agents with a very small probability and impose an extremely high penalty on dishonest agents. This scheme would approximate the complete information case.

\footnotetext{
* We would like to thank Parkash Chander, Luis Corchón, Carmen Herrero, José A. Silva, Fernando Vega-Redondo, and two anonymous referees for helpful suggestions and discussion. We acknowledge financial assistance from the I.V.I.E. and the Spanish Ministry of Education, DGICYT Projects No. PB 93-0940 and PB 94-1504.
} 
Our objective is to characterize the optimal audit probabilities and tax scheme when the penalty function is exogenously given to the principal, but drawn from a large admissible set. To avoid the above kind of situation, we require the admissible functions to satisfy three conditions that we believe are natural in most societies: (i) there is a maximum penalty given by the true income of the agent, (ii) audited dishonest agents pay at least the same amount as an honest agent on the same income level, and (iii) the punishment imposed fits the crime; see Klepper and Nagin (1989) for a non-technical discussion of the tradeoffs between compliance and taxation.

The main technical difficulty in our approach is that we cannot prove the revelation principle, so a general analysis becomes quite intractable. Thus, we concentrate on characterizing the optimal policy among those who tell the truth. However, if we believe that an objective of society is to design policies which induce honest behavior, then the conclusion of our analysis is that an efficient policy cannot be both honest and progressive, i.e., if the optimal policy is to tell the truth, then the average tax is a decreasing function of income.

This approach may be criticized on the grounds that, in reality, taxpayers do not know the auditing policy with enough accuracy. Our result still holds in models where agents have beliefs about the auditing probabilities which do not need to be correct: if the optimal policy induces honesty (and the government knows it) then it must be regressive.

Recent studies on optimal income taxation have addressed this problem in analogous settings; see e.g. Reinganum and Wilde (1985), Border and Sobel (1987) and Mookherjee and Png (1989). Others, such as Sanchez and Sobel (1993) and Cremer, Marchand and Pestieau (1990), characterize the optimal policy for similar models in which a specific penalty function is given for the government. However, as pointed out by Chander and Wilde (1993), the penalty could be more than the true income of a taxpayer.

Chander and Wilde (1993) analyze a similar model. They consider four cases, each associated with a different kind of restriction on the set of admissible penalties (in two of them, some rewards are allowed). None of these penalties is too severe in the sense that the penalty imposed on a dishonest agent cannot be greater than her income. Assuming that the government and the agents are risk neutral, in all four cases they show that the optimal tax scheme induces a concave and non-decreasing "payment function".

One possible objection to the first case studied by Chander and Wilde (1993), i.e., when the penalty function is exogenously given to the government, is that they consider only a very specific penalty function. Moreover, that function is not always a penalty because it allows for cases in which an 
agent who is found to be dishonest ends up paying less taxes than an honest agent with the same initial income. The rest of the cases analyzed by Chander and Wilde, i.e., when the penalty function is endogenously determined, hold for a large set of penalties. However, as the authors show, if the government is free to choose the penalties, the most severe (the so-called "draconian") penalty function will be adopted: whenever an agent is found to be dishonest she will have to pay her total income.

Hence, the penalty assigned to a dishonest agent does not depend on the amount misreported. In most real-life economies, however, there are social norms against this kind of punishment, not only because it is too 'severe", but also because it is not continuous and therefore not in agreement with the principle that "punishment should fit the crime".

\section{The Model}

We consider a population of taxpayers (agents) whose income is distributed along an interval $[0, M]$ according to a measure, $\mu$, on that interval. The measure $\mu$ could be non-atomic or discrete, so we allow for a continuum or a discrete number of agents. We assume that the functions considered below are $\mu$-integrable. We identify the type of agent by her income and let $y \in[0, M]$ denote the agent with income $y$.

The government (principal) collects taxes from the agents, according to a mapping $t:[0, M] \rightarrow \mathbb{R}$ which depends on their (reported) income.

Assumption 1. The tax function $t:[0, M] \rightarrow \mathbb{R}$ satisfies $0 \leq t(x) \leq x$.

Taxpayers observe their income costlessly and they file an income report with the government. The principal knows $\mu$ but it can detect misreporting only by randomly inspecting taxpayers at a cost per inspection of $c>0$. The government announces a random inspection policy according to a certain probability function $p(x):[0, M] \rightarrow[0,1]$ and can credibly commit to it.

The true income of a monitored taxpayer is revealed when she is inspected. Whenever taxpayer $y \in[0, M]$ reports income $x \in[0, M]$ and is not inspected, then the government collects from her the amount $t(x)$. If she reports income $x$ and is audited, then she has to pay the amount $f(x, y)=t(y)+\phi_{t}(x, y)$, where $\phi_{t}(x, y)=\varphi(x, t(x) ; y, t(y))$ is the punishment imposed on agent $y$ in addition to her own taxes.

We assume that the penalty $\phi_{t}$ at most depends only on the type of agent, $y$, the amount she should have paid according to the law, $t(y)$, the type she announces, $x$, and the corresponding tax, $t(x)$. Thus, $\varphi: \mathbb{R}_{+}^{4} \rightarrow \mathbb{R}$ and $\varphi(u, v ; r, s)$ is interpreted as the punishment imposed on agent $r$, whom the law requires to pay $s$ for declaring herself as being of type $u$ and having to pay $v$. Note that we are only fixing a functional form of $f$, i.e., the value of 
$f(x, y)$ might depend on the particular tax function $t$ as well as on $x$ and $y$. To emphasize this dependency we write $t$ explicitly, as a subscript of $\phi$.

Assumption 2. For each $u \geq v, r \geq s$ the following hold:

(a) The mapping $\varphi(u, v ; r, s)$ is continuous, non-decreasing in $r, s$ and nonincreasing in $u$ and $v$.

(b) $0 \leq \varphi(u, v ; r, s) \leq r-s$ and $\varphi(u, v ; u, v)=0$.

It follows that the function $\phi_{t}(x, y)$ satisfies the following properties:

(i) $0 \leq \phi_{t}(x, y) \leq y-t(y)$.

(ii) For $x$ fixed, $\phi_{t}(x, y)$ is non-decreasing in $y$.

(iii) For $y$ fixed, $\phi_{t}(x, y)$ is non-increasing in $x$.

(iv) $\phi_{t}(y, y)=0$.

Condition (i) guarantees that no agent is required to pay more than her true income. It also implies that $\phi_{t}$ is always a penalty, i.e., the amount paid by a monitored dishonest agent is at least the same as what she would have to pay when reporting truthfully.

Whenever two agents misreport the same income, property (ii) requires the one with the greater wealth to pay a higher penalty. According to (iii), the smaller an agent claims her wealth to be, the higher the penalty she would have to pay on inspection. These restrictions may appear unnatural if the tax function $t(x)$ is decreasing. However, in the next section we will see that the optimal tax function and the monitoring probability must both be nondecreasing, even for penalty functions which do not necessarily satisfy (ii) and (iii) above.

Finally, (iv) rules out rewards or punishments for truthfully reporting one's own type. If this property is not assumed, the results in Border and Sobel (1987) suggest that rewards for truthful reporting would be part of the optimal tax system. In view of horizontal equity and since we will only consider "truth-telling" tax policies, property (iv) seems to be a natural assumption.

Thus, given tax system $w=(t, p, f)$, if agent $y$ reports income $x$, her expected taxes will be

$s(x, y)=(1-p(x)) t(x)+p(x) f(x, y)$

and she chooses her report $x$ so as to minimize $s(x, y)$. That is, agents are risk neutral and know the inspection policy $p(x)$.

Our assumptions imply that an agent with income $y$ will not report $z$ if $t(z)>t(y)$. Thus, a rational agent is never required to pay more than her income regardless of whether she is inspected or not. Let us define:

$\alpha(y)=\{z \in[0, M]$ : for each $x \in[0, M], s(z, y) \leq s(x, y)\}$. 
Assumption 3. We only consider tax systems $w=(t, p, f)$, such that for each $y \in[0, M], \alpha(y) \neq \varnothing$.

This assumption holds whenever there is a finite number of agents. The fact that $\alpha(y)$ may contain more than one element will not alter the analysis below. For simplicity, we assume that one element in $\alpha(y)$ is chosen (e.g. take sup $\alpha(y))$ and think of $\alpha$ as being single valued. We also assume that if $y \in \alpha(y)$, then agent $y$ will report honestly.

Hence, given a tax system $w=(t, p, f)$, the expected transfer of money from agent $y$ to the government will be given by $T(y)=s(\alpha(y), y)$ and the net revenue of the government is given by the expression:

$R(w)=\int_{[0, M]}(T(y)-c p(\alpha(y))) \mathrm{d} \mu$.

The penalty function $\varphi$ is imposed exogenously on the government, for example by the legal system or some other institution not contemplated in the model. Given a fixed penalty function $\varphi$, the government chooses a policy, $(t, p)$ satisfying assumptions 1,2 and 3 and such that the tax system $w=(t, p, f)$ maximizes $R(w)$. Hence, the principal is also risk neutral and it is not concerned with distributional issues.

Definition. Given a fixed penalty function $\varphi$, we say that the policy $(t, p)$ is optimal if $R(t, p, f) \geq R(\bar{t}, \bar{p}, f)$ for any other policy $(\bar{t}, \bar{p})$. A tax function $t$ is said to be regressive if $t(x) / x$ is decreasing.

\section{Main Results}

We now turn to a partial characterization of the optimal policy. In contrast with some of the earlier literature, our analysis covers the characterization of the optimal policy for each, arbitrary but fixed, penalty function drawn from a large set of admissible ones. Unless explicitly stated otherwise, we suppose the assumptions made in the preceding section hold.

From (1) we see that agent $y$ will prefer not to declare income $x$ as long as the government inspects type $x$ with probability $p(x)$ greater than

$p(x) \geq \frac{t(y)-t(x)}{f(x, y)-t(x)}=Q(x, t(x) ; y, t(y))$

where, for a fixed penalty function $\varphi$, we define $Q(u, v ; r, s)$ as

$Q(u, v ; r, s)=\left\{\begin{array}{cl}\frac{s-v}{\varphi(u, v ; r, s)+s-v} & \text { if } v \leq s \\ 0 & \text { otherwise }\end{array}\right.$ 
Thus, for each $x, y \in[0, M]$ such that $t(x)<t(y)$, the amount $Q(x, t(x) ; y, t(y))$ measures the "incentives" of agent $y$ to report income $x$. The set of admissible penalty functions we study is given by those which satisfy, in addition to assumption 2, the following:

Assumption 4. The penalty function $\varphi$ satisfies:

(i) If $v \leq u<r \leq r_{0}, s \leq r, v \leq s<s_{0} \leq r_{0}$ and

$\frac{v}{u} \geq \frac{s_{0}}{r_{0}} \geq \frac{s}{r}$

then $Q\left(u, v ; r_{0}, s_{0}\right) \geq Q(u, v ; r, s)$, with strict inequality if $s_{0} / r_{0}>s / r$.

(ii) If $v \leq v_{0}<s$ then $Q(u, v ; r, s) \geq Q\left(u, v_{0} ; r, s\right)$.

In other words, suppose two agents $r_{0}$ and $r$, with $r_{0}>r$, are considering the possibility of reporting themselves as being of type $u<r$, with an obligation to pay $v$. And assume that the mean tax imposed on agent $r_{0}$ (i.e., $s_{0} / r_{0}$ ) is greater than the same quantity for agent $r$ (i.e., $\left.s / r\right)$. Then, assumption 4 (i) implies that it will be more difficult to persuade agent $r_{0}$ than agent $r$ to tell the truth, in the sense that $p(u)$, the probability of inspection, has to be higher in order to dissuade her from misreporting $u$.

On the other hand, assumption 4 (ii) says that, provided $v \leq v_{0}<s$, raising taxes for agent $u$, from $v$ to $v_{0}$ makes reporting $u$ less attractive for any other agent $r>u$.

In order to exemplify the penalty functions satisfying hypotheses 4 above, we begin by examining some penalty functions considered previously in the literature.

Example $A$. The penalty function

$f_{1}(x, y)= \begin{cases}t(x)+y-x, & x \leq y \\ t(y) & x \geq y\end{cases}$

studied in Chander and Wilde (1993), can be obtained from

$\varphi_{1}(u, v ; r, s)=\left\{\begin{array}{ll}v-s+r-u, & r \neq u \\ 0 & r=u\end{array}\right.$.

The penalty function

$f_{2}(x, y)= \begin{cases}t(x)+(1+\pi)(t(y)-t(x)), & x \leq y \\ t(y) & x \geq y\end{cases}$

considered in Sanchez and Sobel (1993), also satisfies assumption 4. Here, $\pi$ is the "penalty rate". 
Example B. Let $0<a \leq 1$ and take $C:[0, \infty) \rightarrow[0, \infty)$ non-decreasing such that $C(\theta) \leq 2 M(\theta-1)$ if $\theta \leq 1$ and $C(\theta)=0$ otherwise (this restriction is imposed to assure that $\varphi$ is non-increasing in $v$ ). The mappings

$\varphi_{3}(u, v ; r, s)= \begin{cases}C(r / s) v^{2}-C(r / s) v s+a(r-s)(1-v / s), & v \leq s \\ 0 & v>s\end{cases}$

correspond to the penalty functions

$f_{3}(x, y)=\left\{\begin{array}{ccc}t(y)+C(y / t(y)) t^{2}(x)-C(y / t(y)) t(x) t(y)+a(y-t(y)) & \\ \times(1-(t(x) / t(y))), & x \leq y \\ t(y) & x>y\end{array}\right.$

which include all those which are linear and quadratic in $t(x)$. Varying $C \geq 0$ changes the convexity of the penalty $f_{3}(x, y)$. For $C=0$, the resulting penalty is linear in $t(x)$, whereas $C$ large corresponds to very convex penalties. Alternatively, the mapping

$f_{4}(x, y)= \begin{cases}t(y)+a(y-x-t(y)+(x / y) t(y)), & x \leq y \\ t(y) & x>y\end{cases}$

also satisfies 4. In this case, the punishment is linear in the amount of tax the agent is trying to evade.

The following result is similar to that of Krasa and Villamil (1994), who study an optimal contracting model with costly verification technology and non-identical, possibly risk averse agents. They show that the transfer mapping, as well as the monitoring probabilities, are decreasing functions of wealth. In their model, "it is optimal to punish an agent (...) by seizing the entire endowment for misreporting" (p. 178). In contrast, we focus on whether qualitatively similar results hold whenever social norms impose economic restrictions on the penalty functions.

Proposition 1. Let $\varphi$ be a penalty function satisfying assumptions $2(b)$ and 4 . Suppose the tax policy $w=(t, p, f)$ is optimal and induces honest reporting. Then, $t$ is non-decreasing and $p$ is non-increasing.

Proof: Suppose $t$ is not non-decreasing and let $y, z \in[0, M]$, such that $z<y$ and $t(z)>t(y)$. Then, $t(z) / z>t(y) / y$ and it follows from assumption 4 (i) that $Q(x, t(x) ; z, t(z))>Q(x, t(x) ; y, t(y))$. Since $(t, p, f)$ induces truthful reporting, we have that

$p(x) \geq \sup _{t(\theta)>t(x)} \frac{t(\theta)-t(x)}{f(x, \theta)-t(x)}=\sup _{t(\theta)>t(x)} Q(x, t(x) ; \theta, t(\theta))$ 
for all $x \in[0, M]$. Let $x \in[0, M]$ such that $t(z)>t(x)$. Then $p(x) \geq Q(x, t(x) ; z, t(z))$ and since $t(z)>t(y)$, we also have that $p(x) \geq Q(x, t(x) ; z, t(z))>Q(x, t(x) ; y, t(y))$. Hence, the incentive constraints for agent $y$ are not binding.

We can raise her taxes by a small amount such that her incentives will continue to be non-binding. This will not change her report and will not induce other agents to revise their reports either. Raising taxes for agent $y$ will yield a higher revenue for the government at the same cost, contradicting the optimality of $(t, p, f)$. Hence, $t$ must be non-decreasing. Since $(t, p, f)$ is optimal, we have that $p(x)=\sup _{t(y)>t(x)} Q(x, t(x) ; y, t(y))$ is nonincreasing.

We can now state the main result. A similar result has already been obtained by Chander and Wilde (1993) for some particular penalty functions. One difference with the approach taken here is that we consider a tax function be regressive if the average tax, rather than the marginal tax, is decreasing. In addition, we restrict ourselves to tax policies which induce truthful reporting since, in the present context, the revelation principle does not necessarily hold.

Theorem. Let $\varphi$ be a fixed penalty function and let $(t, p, f)$ be an optimal tax policy such that assumption 4 holds. Then, either $(t, p, f)$ does not induce truthful reporting, or else $t$ is regressive.

Proof: Given a tax function $t(x)$ define

$t_{*}(x)=x \sup \left\{\frac{t(z)}{z}: z \geq x\right\}$.

Note that $t_{*}(x) / x$ is decreasing and for each $x \in[0, M], t(x) \leq t_{*}(x) \leq x$. The proof of the theorem is now a straightforward consequence of proposition 1 and the following:

Proposition 2. If the tax function $t$ is non-decreasing and the tax system $w=(t, p, f)$ satisfies assumption 4 and induces truthful reporting, then $t_{*}$ is non-decreasing, continuous and $\left(t_{*}, p, f_{t_{*}}\right)$ induces truthful reporting.

Proof: See Appendix.

One possible criticism to the approach above is that agents need to know the inspection rate. We show next that the same result holds, even if the agents have only imperfect information on $p(x)$, as long as the government knows the beliefs of the agents about $p(x)$. Let us modify our model as follows. Taxpayer $y$ may not know the function $p(x)$. Rather, she thinks that the probability of being inspected when she reports her income to be $x$ is given by some probability function $p^{y}(x)$. 
Consequently, given a tax system $w=(t, p, f)$, agent $y$ will not seek to minimize $s(x, y)$ given in equation (1), but she will rather attempt to minimize

$s^{y}(x, y)=t(x)+p^{y}(x)(f(x, y)-t(x))$.

Since the proof of proposition 2 shows that replacing $t(x)$ by $t_{*}(x)$ does not change the incentives of the agents to misreport, our previous reasoning still applies. Of course, nothing could now be said about the true probability of inspection $p(x)$, without further information relating $p(x)$ and $p^{y}(x)$.

Proposition 3. Let $\varphi$ be a penalty function satisfying assumptions 2 and 4 and suppose all the agents have the same beliefs on $p(x)$ (i.e., $p^{y}(x)=p^{z}(x)$ for all $y$ and $z)$, the tax policy $w=(t, p, f)$ is optimal and induces honest reporting. Then, $t$ is non-decreasing and regressive.

One possible scenario in which the analysis above may apply is when agents have some indirect information about the probability of inspection. For example, the number of tax inspectors is public and hence, the total number of inspections which can be carried out, say $n$, can be estimated by the agents. Let $N$ be the number of taxpayers. It is not unreasonable to assume that all agents believe that the probability of being inspected is constant and given by $\bar{p}=n / N$. Even though this may not be the real inspection policy designed by the government, agents have no further information about it. Of course, the beliefs of the agents have to be consistent with the monitoring policy adopted by the government, which has to be able to commit to it. But now the commitment is in the "signal" agents observe and not in the actual probability of inspection. This commitment capacity can be justified by the same arguments used in the standard case where agents observe the true inspection function.

Finally, we examine other alternatives for the government's objective. Recall that in the above discussion the goal of the principal is to find a tax policy which maximizes net revenue $R(w)$ given by equation (2). An alternative approach would be to consider the following problem: given a penalty function $\varphi$, find a tax policy $w=(t, p, f)$ which collects a fixed target revenue, $R(w)$, at a minimum cost.

We argue that if the government restricts itself to honest tax policies and $\mu$ has a continuous density on $[0, M]$, then the optimal tax function for this problem must be regressive as well. Indeed, suppose $\varphi$ satisfies assumption 4 and $w=(t, p)$ is a solution to the problem, which induces truthful reporting. If $t$ is not regressive, we may apply proposition 2 to obtain a new tax function $t_{*}(x)$ which yields a higher revenue at the same cost. Thus, letting $w_{*}=\left(t_{*}, p\right)$, we must have that $R\left(w_{*}\right)>R(w)$. Since $t_{*}$ is continuous and $\mu$ has a continuous density, there is $x_{0} \in[0, M]$ such that 
$\int_{0}^{x_{0}} t_{*}(y) \mathrm{d} \mu+t_{*}\left(x_{0}\right) \mu\left(\left[x_{0}, M\right]\right)=R(w)$.

It is straightforward to verify that the policy

$\bar{t}(x)=\left\{\begin{array}{ll}t_{*}(x) & \text { if } x \leq x_{0} \\ t_{*}\left(x_{0}\right) & \text { if } x \geq x_{0}\end{array}\right.$ and $\quad \bar{p}(x)= \begin{cases}p(x) & \text { if } x \leq x_{0} \\ 0 & \text { if } x \geq x_{0}\end{cases}$

induces truthful reporting and produces the same revenue as $R(w)$ at a lower cost. This proof can easily be adapted to the discrete case.

\section{Final Comments}

In an income tax enforcement model with a principal that has the capability to set the tax and inspection functions, we proved that if the optimal policy is to tell the truth, then: (i) the tax policy is continuous, nondecreasing and regressive, and (ii) the inspection function is non-increasing. Assertion (i) does not depend on whether agents know the true probability of inspection.

These results are related to those of Chander and Wilde (1993). The main difference is that ours apply to any penalty function drawn from a large set of penalties satisfying certain "social norms". Thus, the model and the reasoning behind the proofs change substantially. The intuitive explanation of Chander and Wilde's results, based on the specific penalty functions they consider, is that in an optimal policy, the audit probability at any point equals the marginal tax rate of the agent in question.

In order to explain the intuition in our model, suppose there are only three types of agents $x<y<z$, and suppose the government has implemented a certain tax policy. Our assumptions imply that if $t(z) / z>t(y) / y$, then the incentives for $z$ to report $x$ are higher than the incentives for $y$ to file the same report. Thus, provided the tax policy designed induces honest reporting, the probability of inspection $p(x)$ cannot be binding for agent $y$. It follows that the government can now increase the tax for agents with rent $y$ and the tax policy we started with could not possibly be optimal.

The introduction of risk averse agents, as in Krasa and Villamil (1994), additional information about the taxpayer and the study of dishonest optimal policies are important topics for further research.

\section{Appendix. Proof of Proposition 2}

Let $x<y$ and suppose $t_{*}(x)>t_{*}(y)$. Then we can find $x_{0} \geq x$ such that for each $z \geq y$ 
$x \frac{t\left(x_{0}\right)}{x_{0}}>y \frac{t(z)}{z}$.

Taking $z=y$ we obtain $x t\left(x_{0}\right) / x_{0}>t(y)$. But this is impossible since $x / x_{0} \leq 1$ and $t\left(x_{0}\right) \leq t(y)$. Hence, $t_{*}$ is non-decreasing.

Let $x \leq y$. We show next that $t_{*}(x)$ does not change the incentives for $y$. It is enough to prove that $p(x) \geq Q\left(x, t_{*}(x) ; y, t_{*}(y)\right)$.

First, if $t(y)=t_{*}(y)$, then applying assumption 4 (ii) and taking into account that $t(x) \leq t_{*}(x)$ and $t$ induces truthful reporting, we have $p(x) \geq Q(x, t(x)$; $y, t(y))>Q\left(x, t_{*}(x) ; y, t_{*}(y)\right)$ so $y$ prefers not to declare herself as being of type $x$.

Otherwise, $t(y)<t_{*}(y)$. Take a sequence $\left\{a_{n}\right\}_{n=1}^{\infty}$ contained in $(y, M)$ and such that

$\frac{t\left(a_{n}\right)}{a_{n}}>\frac{t(y)}{y}$ and $\lim _{n \rightarrow \infty} \frac{t\left(a_{n}\right)}{a_{n}}=\frac{t_{*}(y)}{y}$.

It follows that for each $n \in \mathbb{N}, t\left(a_{n}\right) \geq t(y)$. By a compactness argument, we may assume the sequence converges to, say, $z \in[y, M]$. Thus, $\left\{t\left(a_{n}\right)\right\}_{n=1}^{\infty}$ also converges and

$\lim _{n \rightarrow \infty} t\left(a_{n}\right)=z \frac{t_{*}(y)}{y} \leq z$.

Since $(t, p, f)$ induces truthful reporting, for each $n \in \mathbb{N}$, we have $p(x) \geq Q\left(x, t(x) ; a_{n}, t\left(a_{n}\right)\right)$. Taking limits we obtain that

$p(x) \geq Q\left(x, t(x) ; z, z \frac{t_{*}(y)}{y}\right.$.

We now apply assumption 4 (ii) with $u=x, v=t(x), r=z, s=z t_{*}(y) / y, v_{0}=t_{*}(x)$ to obtain:

$Q\left(x, t(x) ; z, z \frac{t_{*}(y)}{y}\right) \geq Q\left(x, t_{*}(x) ; z, z \frac{t_{*}(y)}{y}\right)$.

We then use 4 (i) with $u=x, v=t_{*}(x), r=y, s=t_{*}(y), r_{0}=z, s_{0}=z t_{*}(y) / y$.

So $t_{*}(x) \leq x<y \leq z, t_{*}(y) \leq y, z t_{*}(y) / y \leq z$ and

$\frac{v}{u}=\frac{t_{*}(x)}{x} \geq \frac{s_{0}}{r_{0}}=\frac{t_{*}(y)}{y}=\frac{s}{r}$.

Hence,

$p(x) \geq Q\left(x, t_{*}(x) ; z, z \frac{t_{*}(y)}{y}\right) \geq Q\left(x, t_{*}(x) ; y, t_{*}(y)\right)$ 
and it does not pay for agent $y$ to declare herself as $x$. Since $x \leq y$ was arbitrary, we see that $\left(t_{*}, p\right)$ also induces truthful reporting.

We show that $t_{*}$ is continuous. Otherwise, since $t_{*}$ is non-decreasing, it can have only jump discontinuities, i.e., if $t_{*}$ is discontinuous at $x_{0}$ then

$$
\lim _{x \rightarrow x_{0}^{-}} t_{*}(x)<\lim _{x \rightarrow x_{0}^{+}} t_{*}(x)
$$

so

$$
\lim _{x \rightarrow x_{0}^{-}} \frac{t_{*}(x)}{x_{0}}<\lim _{x \rightarrow x_{0}^{+}} \frac{t_{*}(x)}{x_{0}} .
$$

But then $t_{*}(x) / x$ is increasing at $x_{0}$.

\section{References}

Border, K. and Sobel, J. P: Samurai accountant: A theory of auditing and plunder. Review of Economic Studies 54, 525-40, 1987.

Chander, P. and Wilde, L.: A general characterization of optimal income taxation and enforcement. Caltech Social Science WP 796, 1993.

Cremer, H., Marchand, M. and Pestieau, P.: Evading auditing and taxing: The equity-compliance tradeoff. Journal of Public Economics 43, 67-92, 1990.

Klepper, S. and Nagin, D.: The criminal deterrence literature. In J. A. Roth, J. T. Scholz and A. D. Witte (eds.), Taxpayer Compliance, Vol. 2, University of Pennsylvania Press, 1989.

Krasa, S. and Villamil, A. P.: Optimal multilateral contracts. Economic Theory 4, 167-87, 1994.

Mookherjee, D. and Png, I.: Optimal auditing, insurance and redistribution. Quarterly Journal of Economics 104, 399-415, 1989.

Reinganum, J. F. and Wilde, L. L.: Income tax compliance in a principal-agent framework. Journal of Public Economics 26, 1-18, 1985.

Sanchez, I. and Sobel, J.: Hierarchical design and enforcement of income tax policies. Journal of Public Economics 50, 345-69, 1993.

First version submitted February 1995;

final version received May 1996. 